\title{
Proton temperature anisotropy in the magnetosheath: comparison of 3-D MHD modelling with Cluster data
}

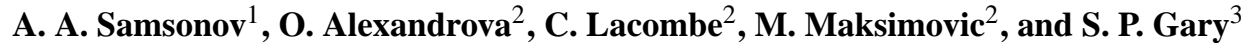 \\ ${ }^{1}$ Institute of Physics, St. Petersburg State University, St. Petersburg, Russia \\ ${ }^{2}$ LESIA/CNRS, Observatoire de Paris, Meudon, France \\ ${ }^{3}$ Los Alamos National Laboratory, Los Alamos, New Mexico, USA
}

Received: 2 November 2006 - Revised: 20 February 2007 - Accepted: 4 April 2007 - Published: 4 June 2007

\begin{abstract}
We study four intervals of Cluster data, lasting from five to eight hours, in the flanks of the magnetosheath. In a first part, we make numerical simulations of these magnetosheath crossings, using a three-dimensional double-adiabatic MHD model of the magnetosheath and assuming that the proton temperature anisotropy is bounded by the kinetic thresholds of the Alfvén proton cyclotron instability and of the mirror instability. The conditions at the upstream boundary of the numerical domain are given by the solar wind parameters observed by ACE. We assume that the magnetopause is a fixed and impenetrable boundary, i.e. without magnetic reconnection. The global agreement between the observations and the simulations confirms the validity of the model in the magnetosheath flanks. We discuss the consequences of different models of the magnetopause on some simulation results. In a second part, we compare the observed proton temperature anisotropy and the kinetic anisotropy thresholds of the two above-mentioned instabilities which are local functions of the proton $\beta$. In the intervals with a low proton $\beta$, the observed temperature anisotropy agrees well with the kinetic threshold of the proton-cyclotron instability; in the intervals with a higher $\beta$, the observed anisotropy is close to both the proton-cyclotron and the mirror thresholds. This confirms that the observed proton anisotropy is indeed bounded by the instability thresholds. We then analyse the magnetic field power spectra in a frequency range $0.003-10 \mathrm{~Hz}$ during four 18 -min intervals for different values of $\beta$. If $\beta<1$, transverse (i.e. Alfvénic) fluctuations are dominant at every frequency. For $\beta \geq 1$, a mixture of compressive (i.e. mirror) and transverse waves is usually observed. For a case with $\beta \simeq 10$, there is no frequency where compressive waves are dominant. The values of $\beta$ and of the proton temperature anisotropy are thus important but not the only parameters which determine the domi-
\end{abstract}

Correspondence to: A. A. Samsonov

(samsonov@geo.phys.spbu.ru) nant mode, compressive or transverse, at the proton scales in the magnetosheath.

Keywords. Magnetospheric physics (Magnetosheath; Plasma waves and instabilities; Solar wind-magnetosphere interactions)

\section{Introduction}

The magnetosheath is a region between the bow shock and the magnetopause where the plasma is hotter and more compressed than in the upstream solar wind. This is a nice natural laboratory for studying plasma turbulence. There are several sources of plasma waves and instabilities in the magnetosheath: some of the waves come from the upstream solar wind or originate at the bow shock, some may appear at the magnetopause (for example, due to the magnetic reconnection or surface waves). Finally, a third possible source of the waves and instabilities is inside the magnetosheath and is related to the particle temperature anisotropy. As it was found first in Explorer 33 measurements (Crooker et al., 1976), the proton temperature perpendicular to the magnetic field $T_{\perp p}$ is generally larger than the parallel temperature $T_{\| p}$ in the dayside magnetosheath.

The linear kinetic theory predicts that two electromagnetic ion temperature anisotropy instabilities, proton cyclotron and mirror, may grow under the condition $T_{\perp p}>T_{\| p}$ (Gary, 1993). Both of them have been identified many times in the magnetosheath, at proton scales, i.e. at wavelengths equal to about ten times the thermal proton gyroradius (e.g. Tsurutani et al., 1982; Hubert et al., 1989; Anderson and Fuselier, 1993; Song et al., 1994; Lacombe and Belmont, 1995; Czaykowska et al., 1998, 2001; Hubert et al., 1998; Lucek et al., 2001; Sahraoui et al., 2003; Alexandrova et al., 2004; Schäfer et al., 2005). However, such an identification is often a complicated problem, and a comprehensive wave analysis

Published by Copernicus GmbH on behalf of the European Geosciences Union. 
reveals a mixture of different wave modes in most observed events.

Relations between the thresholds of the two instabilities, their maximum growth rates, and the plasma beta have been found using the linear kinetic theory (e.g. Gary, 1993; Gary et al., 1994a; Hellinger et al., 2006). An analytical approach of the mirror instability, accounting for arbitrarily large ion-Larmor radius effects, is developed by Pokhotelov et al. (2004). Gary et al. (1994a) show that the levels of constant growth rate in the plane $\left(\beta_{\| p}, T_{\perp p} / T_{\| p}\right)$ obey the following relation:

$T_{\perp p} / T_{\| p}=1+S_{p} \beta_{\| p}^{-\alpha_{p}}$

where $S_{p}$ and $\alpha_{p}$ are coefficients which depend on the considered instability and on a particular level of the growth rate. This kind of relation between $T_{\perp p} / T_{\| p}$ and $\beta_{\| p}$ determines an upper limit of the observed $T_{\perp p} / T_{\| p}$ in the magnetosheath data (Anderson et al., 1994; Fuselier et al., 1994; Phan et al., 1994, 1996).

Chew et al. (1956) in their classical double-adiabatic model (CGL model) proposed to calculate separately variations of the perpendicular and parallel temperatures arising from the magnetic field/plasma compression and expansion. The CGL model was converted into a bounded anisotropy model (BAM) by Denton et al. (1994), who used Eq. (1) as an upper limit of $T_{\perp p} / T_{\| p}$ in the magnetosheath, in agreement with observations. When the ratio $T_{\perp p} / T_{\| p}$ exceeds this limit, the development of the anisotropy instabilities results in an energy transformation from the perpendicular to parallel degrees of freedom. The theoretical model of Denton et al. has stimulated development of numerical anisotropic MHD models which use Eq. (1) for the limitation of the anisotropy. Denton and Lyon (1996) presented a simplified two-dimensional (2-D) MHD model, but more realistic threedimensional (3-D) MHD models were developed by Samsonov and Pudovkin (1998), Erkaev et al. (1999) and Samsonov and Pudovkin (2000). A comparison of profiles of the MHD parameters in the subsolar region for the anisotropic and isotropic MHD models has shown that the difference between them would be usually no more than 10-15 percent. In particular, Samsonov and Pudovkin (2000) found that the magnetosheath width along the Sun-Earth line, calculated with the anisotropic model, is about 1.14 times larger than the magnetosheath width in the isotropic model. Farrugia et al. (2001) found that the numerical results of the anisotropic model reproduce well the observations of the Wind spacecraft near the stagnation streamline.

The above-mentioned numerical models following the approach of Denton et al. (1994) have used the threshold of the anisotropy derived from experimental data, taking the coefficients in Eq. (1) as $S_{p}=0.85$ and $\alpha_{p}=0.48$. Then a diffusion (or energy exchange) term has been added in the MHD equations which describes the time evolution of $T_{\perp}$ and $T_{\|}$(see Eq. (8) of Denton et al., 1994). Samsonov et al. (2001) have proposed to include three diffusion terms in a similar equation of evolution of $T_{\perp} / T_{\|}$: two terms describe the pitch-angle diffusion due to the mirror and protoncyclotron instabilities, and the third term works only near the bow shock, where the diffusion (and growth of the anisotropy instabilities) seems to be more intensive than in the magnetosheath proper. The thresholds related with the action of the anisotropy instabilities have been obtained by Samsonov et al. (2001) from the linear kinetic theory.

In this paper, we use a 3-D anisotropic MHD model, treating the proton temperature anisotropy in the magnetosheath similar to the model of Samsonov et al. (2001), in order to simulate four intervals of several hours observed by Cluster in the magnetosheath flanks. In the next section, the data are described. The numerical model is explained in Sect. 3. The inner boundary conditions of the simulation imply that there is no magnetic reconnection at the magnetopause. Results of the numerical simulations are presented and discussed in Sect. 4. In Sect. 5, we compare the observed proton temperature anisotropy with theoretical thresholds of the protoncyclotron and mirror instabilities used in the MHD modelling and analyse the role of the two instabilities in the limitation of the proton anisotropy. A few observed spectra of the magnetic field power, shown in Sect. 6, allow one to present a discussion about the dominant wave mode (compressive or transverse) in relation to the previous analysis, using the thresholds of the instabilities. The last section contains the conclusions.

\section{Data}

The data of Cluster 1 (Rumba), in the magnetosheath, are analysed during four intervals. Figure 1 displays the position of Cluster in the GSE planes (X,Y) and (Y,Z) for each interval. A paraboloidal bow shock model without aberration, $X=14.6\left[1-\left(Y^{2}+Z^{2}\right) / 25.6^{2}\right]$ (Filbert and Kellogg, 1979), and a magnetopause model (dashed line; Sibeck et al., 1991), $Y^{2}+Z^{2}=139.2-0.18 X^{2}-14.2 X$ are also shown. The considered intervals are generally far from the bow shock, except the longest interval, on 12 February 2001, which corresponds to a nearly complete crossing of the magnetosheath. The date and the duration of the four intervals, lasting from $5 \mathrm{~h}$ to $8 \mathrm{~h}$, are given in the caption of Fig. 1.

The density, the velocity and the temperatures (Rème et al., 1997) are the successive $(0,1,2)$ moments, computed onboard every $4 \mathrm{~s}$, of the ion distribution function measured by the CIS2(HIA) instrument, without mass separation but with the best time and velocity space resolution. A comparison with densities obtained by the CIS1(CODIF) instrument indicates that the major ion species is $\mathrm{H}^{+}$, with density ratios $n\left(\mathrm{He}^{++}\right) / n\left(\mathrm{H}^{+}\right)$generally smaller than $6 \%$, and $n\left(\mathrm{He}^{+}, \mathrm{O}^{+}\right) / n\left(\mathrm{H}^{+}\right)$generally smaller than $2 \%$. We use $4-\mathrm{s}$ averages of the magnetic field given by the FGM experiment; 

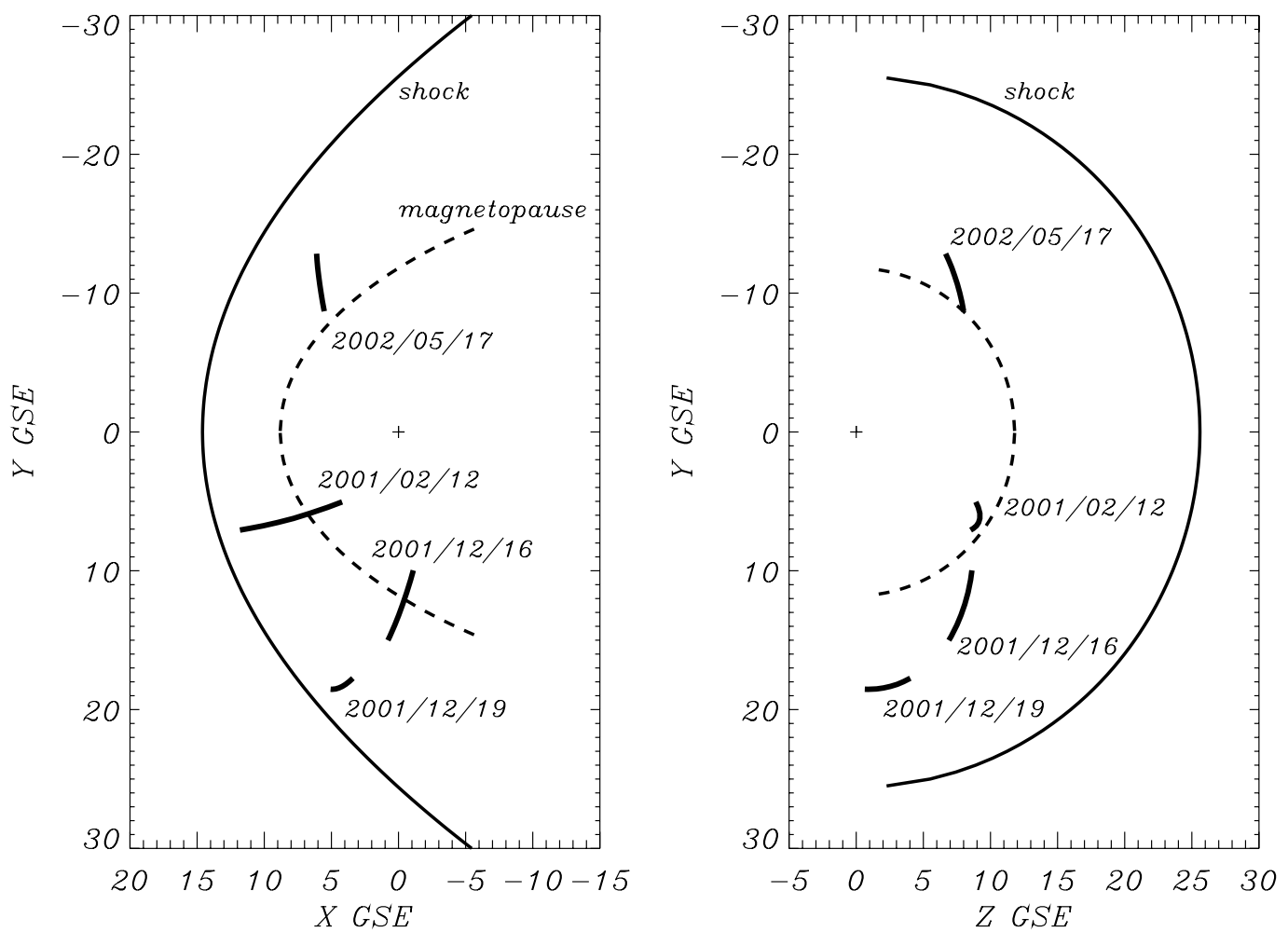

Fig. 1. For the four considered time intervals, projection of the Cluster orbit in the $\left(X_{\mathrm{GSE}}, Y_{\mathrm{GSE}}\right)$ and $\left(Z_{\mathrm{GSE}}, Y_{\mathrm{GSE}}\right)$ planes. Days 12 February 2001 (00:00 to 08:00 UT), 16 December 2001 (03:00 to 09:00 UT), 19 December 2001 (00:00 to 06:00 UT), 17 May 2002 (08:00 to 13:00 UT).

for the spectral analysis of Sect. 6 we use the high resolution FGM data (20 samples per second) (Balogh et al., 1997).

We also use the ACE MAG magnetic field data with 16-s resolution and the ACE SWEPAM plasma data with 64-s resolution measured near the Lagrangian point $\left(X_{\mathrm{GSE}}\right.$ is about $240 R_{E}$ ) as input solar wind parameters for the numerical model. In the same four intervals, the properties of the turbulence at electron scales in the magnetosheath, as functions of the ACE solar wind parameters, have been analysed in the works of Mangeney et al. (2006) and Lacombe et al. (2006).

\section{Numerical model}

This work develops a method of the anisotropic MHD modelling proposed by Samsonov et al. (2001), and Samsonov and Meister (2002). They assumed that there are two independent theoretical thresholds, expressed in the form of Eq. (1), for the mirror and proton-cyclotron instabilities in the magnetosheath.

The following one-fluid 3-D MHD equations deduced from the equations of the bounded anisotropy model of Denton et al. (1994) have been used

$$
\begin{aligned}
& \partial \boldsymbol{B} / \partial t=\nabla \times[\boldsymbol{V} \times \boldsymbol{B}], \\
& \partial \rho / \partial t=-\nabla \cdot(\rho \boldsymbol{V}),
\end{aligned}
$$

$$
\begin{gathered}
\frac{\partial}{\partial t}(\rho \boldsymbol{V})=-\nabla \cdot\left(\rho \boldsymbol{V} \boldsymbol{V}+\mathbf{I}\left(p_{\perp}+\frac{B^{2}}{8 \pi}\right)\right. \\
\left.+\frac{\boldsymbol{B} \boldsymbol{B}}{4 \pi}\left(4 \pi \frac{p_{\|}-p_{\perp}}{B^{2}}-1\right)\right) \\
\partial e / \partial t=-\nabla \cdot \boldsymbol{q}, \quad e=\frac{\rho V^{2}}{2}+\frac{B^{2}}{8 \pi}+p_{\perp}+\frac{1}{2} p_{\|} \\
\boldsymbol{q}=\boldsymbol{V}\left(\frac{\rho V^{2}}{2}+2 p_{\perp}+\frac{1}{2} p_{\|}\right)+\left(p_{\|}-p_{\perp}\right) \boldsymbol{V}_{\|} \\
+\frac{1}{4 \pi}[\boldsymbol{B} \times[\boldsymbol{V} \times \boldsymbol{B}]] \\
\frac{\partial A}{\partial t}=-\nabla \cdot(A \boldsymbol{V})+3 A(\boldsymbol{b} \cdot(\boldsymbol{b} \cdot \nabla) \boldsymbol{V}) \\
+(1+2 A) \frac{1}{p_{\|}}\left(\frac{\partial p_{\perp}}{\partial t}\right)_{\mathrm{dif}}
\end{gathered}
$$

where $A \equiv p_{\perp} / p_{\|}, \boldsymbol{b} \equiv \boldsymbol{B} / B,\left(\partial p_{\perp} / \partial t\right)_{\operatorname{dif}}=\left(\partial p_{\perp} / \partial t\right)_{\operatorname{dif} 1}+$ $\left(\partial p_{\perp} / \partial t\right)_{\operatorname{dif} 2}$.

The two diffusion terms in $\left(\partial p_{\perp} / \partial t\right)_{\text {dif }}$ are related to the proton-cyclotron and mirror instabilities, respectively:

$\left(\frac{\partial p_{\perp}}{\partial t}\right)_{\text {dif1 }}= \begin{cases}-\frac{p_{\perp}}{\tau_{p c}}\left(\frac{p_{\perp}}{p_{\|}}-A_{p c}\right), & \text { if }\left(\frac{p_{\perp}}{p_{\|}}\right) \geq A_{p c}, \\ 0, & \text { if }\left(\frac{p_{\perp}}{p_{\|}}\right)<A_{p c},\end{cases}$ 


$$
\left(\frac{\partial p_{\perp}}{\partial t}\right)_{\text {dif } 2}= \begin{cases}-\frac{p_{\perp}}{\tau_{m i r}}\left(\frac{p_{\perp}}{p_{\|}}-A_{m i r}\right), & \text { if }\left(\frac{p_{\perp}}{p_{\|}}\right) \geq A_{\text {mir }}, \\ 0, & \text { if }\left(\frac{p_{\perp}}{p_{\|}}\right)<A_{\text {mir }} .\end{cases}
$$

Samsonov et al. (2001) and Samsonov and Meister (2002) used the proton-cyclotron and mirror thresholds, $A_{p c}$ and $A_{\text {mir }}$, which correspond to the maximum growth rate $\gamma_{m} / \Omega_{p}=10^{-4}$ in the linear kinetic theory; and they tested a few different time parameters $\tau_{p c}$ and $\tau_{\text {mir }}$ between 100 and $10^{3} \mathrm{~s}$. In this work, we use the thresholds for a larger maximum growth rate $\gamma_{m} / \Omega_{p}=10^{-2}$, which was shown to be the functions of $\beta_{\|}$(Samsonov et al., 2001):

$A_{p c}=1+0.64 \beta_{\|}{ }^{-0.41}$

for the proton-cyclotron instability and

$A_{\text {mir }}=1+0.99 \beta_{\|}^{-0.63}$

for the mirror instability. $\Omega_{p}$ is the proton cyclotron frequency. The maximum growth rate $\gamma_{m}$ has been determined for fixed plasma parameters but for all possible wave numbers and wave vector directions. For the proton-cyclotron instability, the maximum growth rate is always at $k_{\perp}=0\left(k_{\perp}\right.$ is the wave vector perpendicular to the mean magnetic field), but for the mirror mode, the search for the maximum growth rate requires more effort, because one must search in both $k_{\|}$ (parallel wave vector) and $k_{\perp}$. As we will see in Sect. 5, the values $\gamma_{m} / \Omega_{p}=10^{-4}$ and $\gamma_{m} / \Omega_{p}=10^{-2}$ describe well the magnetosheath observations.

We assume that the thresholds (9) and (10) determine an upper limit of a possible $p_{\perp} / p_{\|}$in the magnetosheath, using the conditions $p_{\perp} / p_{\|} \leq A_{p c}$ and $p_{\perp} / p_{\|} \leq A_{\text {mir }}$. It is equivalent to take $\tau_{p c}$ and $\tau_{\text {mir }}$ equal to zero in Eqs. (7) and (8). Thus we consider no dependence on $\tau_{p c}$ and $\tau_{\text {mir }}$ in the present work. We do not use the last term $\left(\partial p_{\perp} / \partial t\right)_{\operatorname{dif} 3}$ of Samsonov et al. (2001) because most profiles, except one, contain no bow shock crossing.

The numerical method used in this work is less diffusive than the one used in Samsonov et al. (2001). Here we use the TVD Lax-Friedrichs II-order scheme (e.g. Tóth and Odstrcil, 1996) for the numerical simulation of the interaction of a supersonic solar wind with a magnetopause obstacle. The numerical dissipation is introduced by the minmod slope limiter. The magnetic field is corrected after a few time steps by the projection scheme to hold the constraint $\operatorname{div}(\boldsymbol{B})=0$. We extend the numerical box to the magnetosheath flanks using the parabolic coordinates apart from the subsolar region, and the spherical coordinates in the subsolar region, in order to avoid the singularity of the parabolic coordinates at the Sun-Earth line. The two coordinate systems intersect and the values from the inner points of one coordinate system have been used to determine the boundary conditions for the other system. The grid spacing increases from about $0.25 R_{E}$ in the subsolar region to about $1 R_{E}$ on the nightside magnetosheath flanks. An illustration of the numerical grid has been shown in Fig. 1 of Samsonov et al. (2006).
The outer boundary of the numerical box is in the supersonic solar wind near the bow shock. We assume that $p_{\perp} / p_{\|}=1$ at this boundary. Usually $p_{\|}$is slightly higher than $p_{\perp}$ in the supersonic solar wind near the Earth's orbit, but we think that taking into account this difference would not significantly change the results in the magnetosheath. The other conditions at the outer boundary are obtained from the ACE magnetic field and plasma measurements, taking into account the time delay between ACE and the bow shock. The resolution of the input parameters is taken to be $16 \mathrm{~s}$, making a linear interpolation of the initial 64-s density, velocity components and proton temperature. For each interval of the magnetosheath data, we calculate the average time delay between ACE and Cluster which gives the best correlation between the magnetic field components on ACE and Cluster. These delays, determined by large IMF discontinuities, vary between 54 and $72 \mathrm{~min}$, i.e. about $1 \mathrm{~h}$ for the four considered intervals.

The inner boundary corresponds to the magnetopause where the physical boundary conditions are $V_{n}=0$ and $B_{n}=0$. There is also an outflow boundary where the plasma leaves the numerical domain with a supersonic velocity, and therefore we use free boundary conditions. Despite some variations of the solar wind dynamic pressure in the intervals, we assume that the magnetopause is a fixed, impenetrable boundary with an average size and shape close to those predicted by Shue et al. (1998), which does not react to the upstream temporal variations. Another approach developed by Samsonov and Hubert (2004) is to take an empirical magnetopause model and to change the distance from the Earth to the subsolar point (i.e. in fact the spatial normalization) in response to changes in the solar wind conditions. In this work, we have made a few runs of simulation testing assumptions about both a fixed magnetopause and a moving magnetopause when the position of subsolar point is determined by the models of Pudovkin et al. (1998), Shue et al. (1998) and pressure balance models. However, only results of the first run with a fixed magnetopause will be shown in the figures. We shall discuss the differences in these approaches in the next section.

\section{Numerical results}

Figures 2 to 5 show the temporal variations of the main MHD variables obtained in the numerical simulation (red lines) in comparison with Cluster data (black lines) for these four intervals. The time-shifted input solar wind parameters are shown by blue lines. Mean values of the fast Mach number $M_{f}$, of the Alfvén Mach number $M_{A}$, of the plasma beta $\beta$ and of the IMF $B_{z}$ component in GSM coordinates, determined from ACE data near the Lagrangian point for each event, are given in Table 1 . These values are typical for a quiet solar wind. Now let us consider each interval separately. 

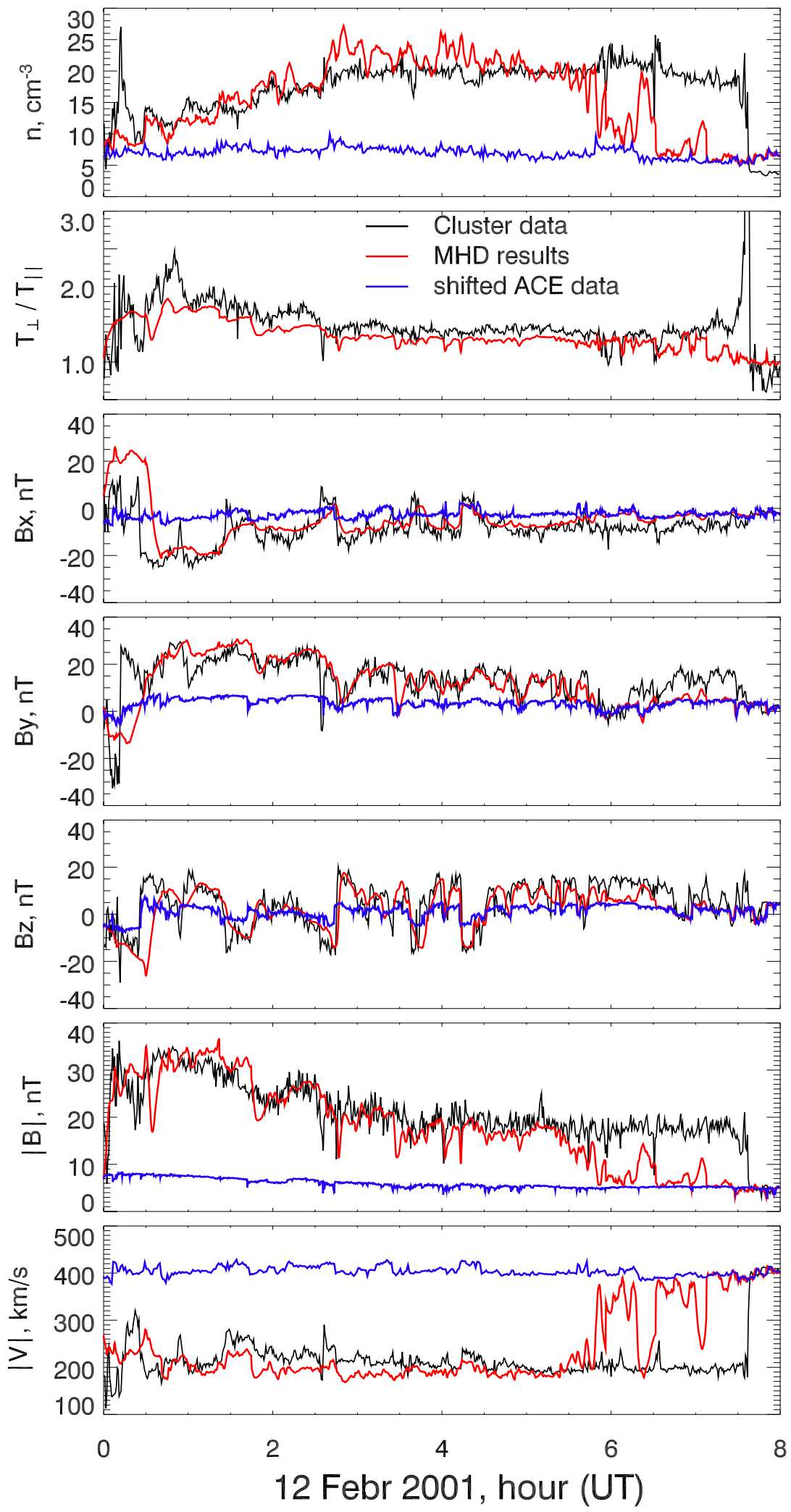

Fig. 2. Temporal variations of MHD parameters in the first interval. The data are shown by black lines, the numerical results are shown by red lines, the time-shifted solar wind data are shown by blue lines. 

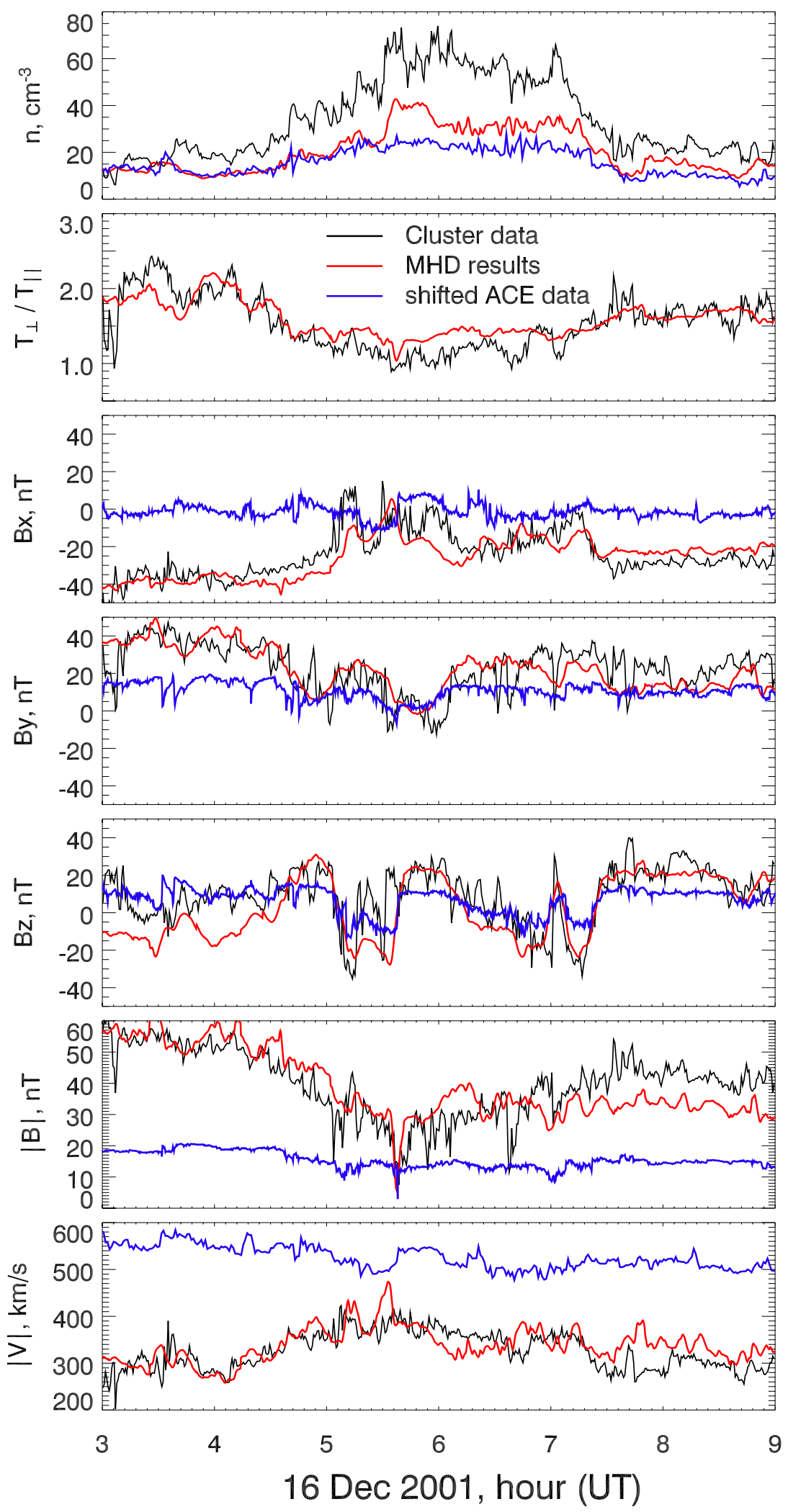

Fig. 3. Temporal variations of MHD parameters in the second interval. The data are shown by black lines, the numerical results are shown by red lines, the time-shifted solar wind data are shown by blue lines. 

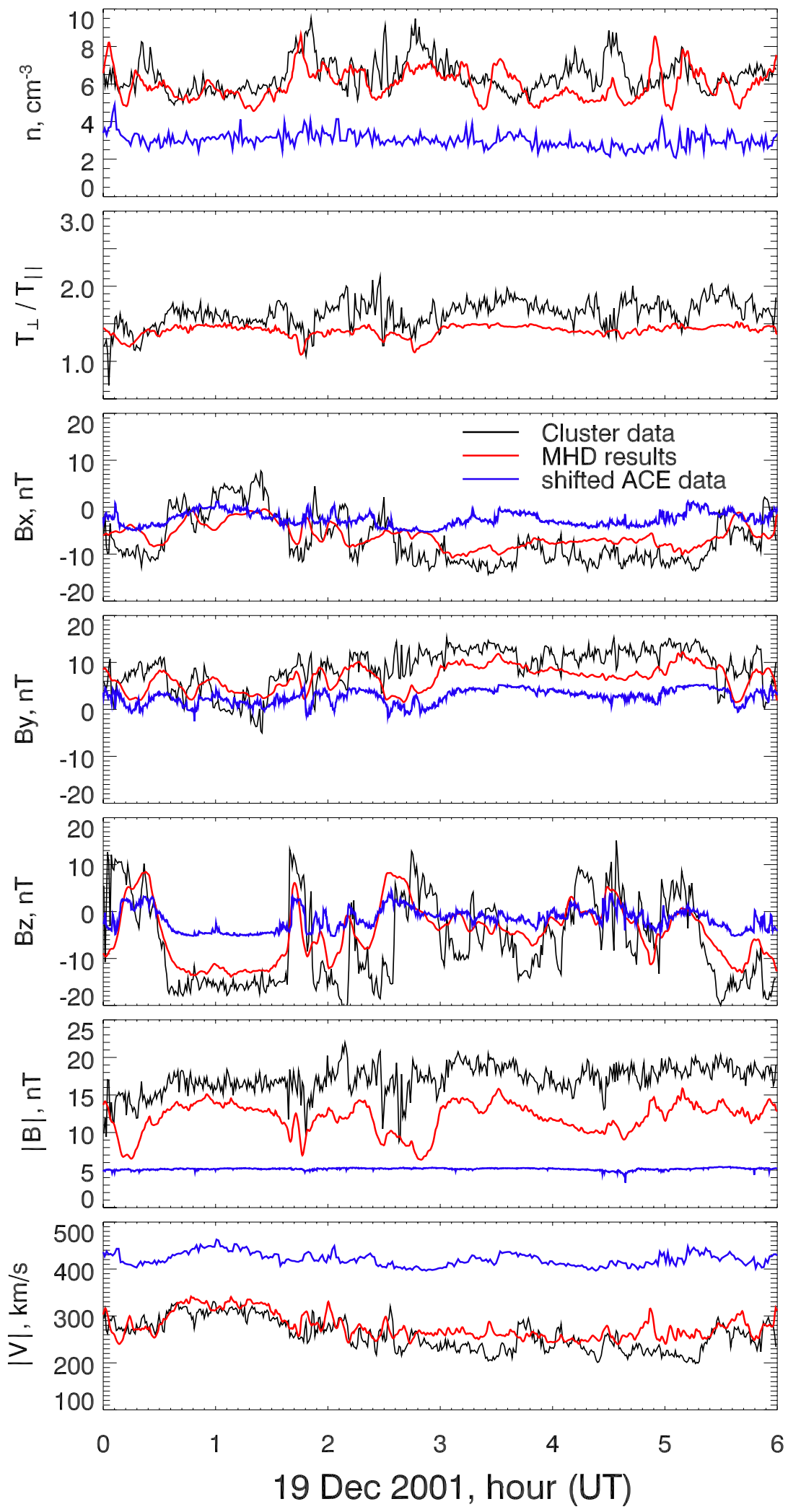

Fig. 4. Temporal variations of MHD parameters in the third interval. The data are shown by black lines, the numerical results are shown by red lines, the time-shifted solar wind data are shown by blue lines. 

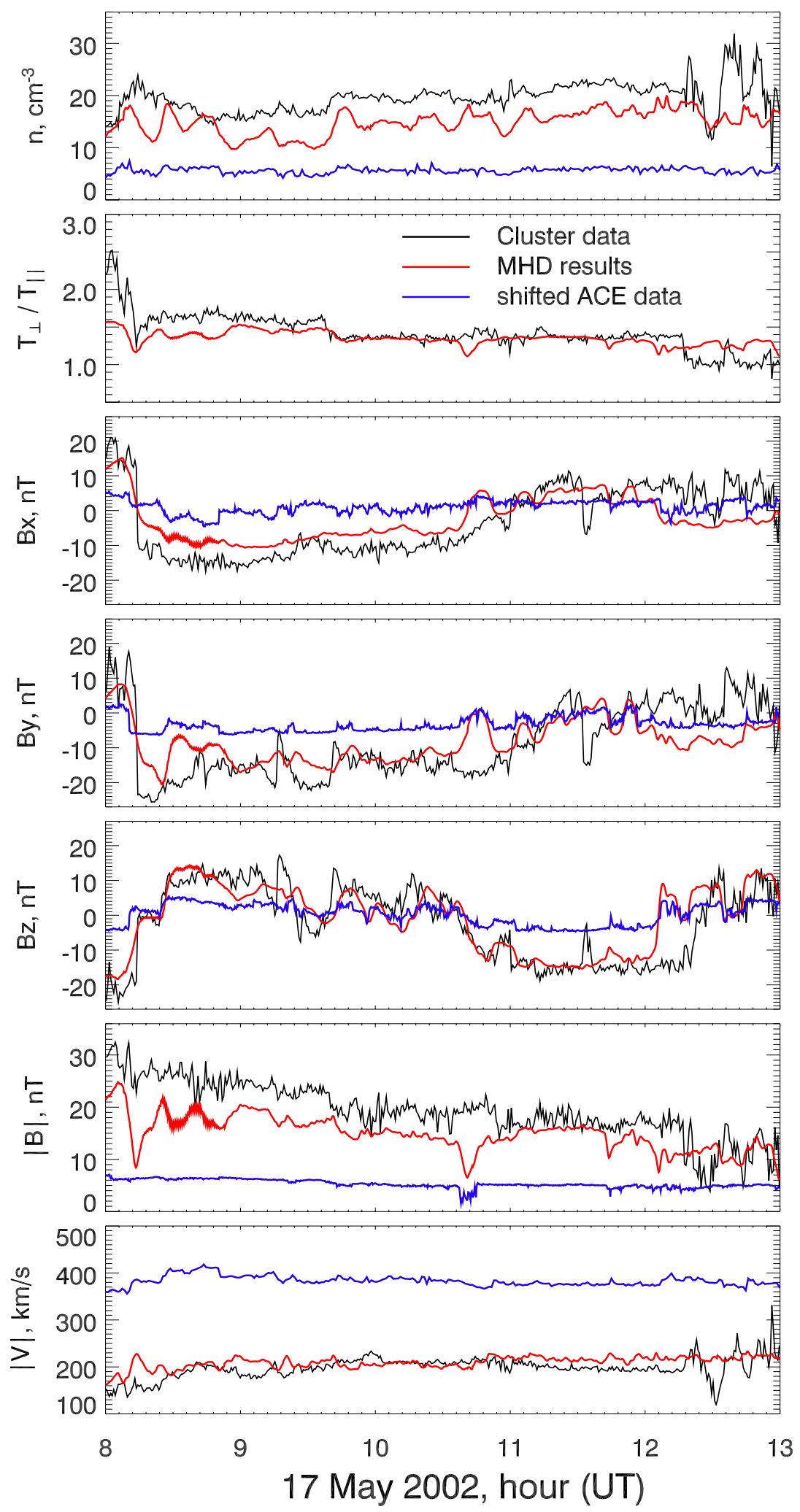

Fig. 5. Temporal variations of MHD parameters in the fourth interval. The data are shown by black lines, the numerical results are shown by red lines, the time-shifted solar wind data are shown by blue lines. 
On 12 February 2001 (Fig. 2), the beginning of the interval is very disturbed and there is a large data gap on the previous day. We think that at midnight the spacecraft is near the magnetopause and may be in the vicinity of the cusp. In the first two hours, the density and $\beta$ (the observed $\beta_{\|}$for these intervals will be shown in Figs. 6 to 9) are smaller, and $|B|$ and $T_{\perp} / T_{\|}$are larger than the average values over the interval. These are typical properties of the plasma depletion layer (PDL) which usually forms in the magnetosheath near the dayside magnetopause. The bow shock crossing is at the end of the interval at 07:36 UT. An almost complete crossing from the magnetopause to the bow shock is thus observed.

Figure 2 shows that generally the simulation reproduces well the data, except that the position of the bow shock is predicted closer to the Earth than it is really observed. A reason for this discrepancy will be discussed below. Average values of the predicted density and magnetic field components are rather close to the observed ones, the predicted $T_{\perp} / T_{\|}$and $|V|$ are a little smaller than in the data. Most variations of the magnetic field orientation are predicted, although small errors exist due to the average time delay used for the convection time of the IMF discontinuities.

Usually numerical results of the local magnetosheath MHD models (e.g. Farrugia et al., 2001; Samsonov and Hubert, 2004) have reproduced magnetosheath crossings in the dayside region. Here we present the results of the numerical simulation on the magnetosheath flanks. Making numerical predictions on the flanks is a more difficult problem than to simulate only the subsolar region. In the subsolar region, the numerical model must reproduce correctly the jumps across the bow shock and the magnetic barrier formation near the magnetopause: this is relatively easy using a conservative form of the 3-D MHD equations. On the contrary, the model must determine well a size and a shape of the magnetopause for correct predictions on the flanks. This determination is rather difficult for a local magnetosheath model, and it may also be a source of errors in predictions of global MHD models.

We may define roughly two types of discrepancies between numerical predictions and observations. First, the average numerical values may differ from the observed ones in a whole interval. Occasionally some discrepancies may be explained by different calibrations of instruments on two spacecraft. However, excluding this possibility, we have to conclude that the difference between the average values results from some model defects and, in particular, is explained by an incorrect approximation of the magnetopause shape. The last is often a fault of local magnetosheath models, because even using an axis-symmetrical (with respect to the Sun-Earth line) magnetopause obstacle is a possible source of mistakes. As it is explained in the previous section, we have made several runs of the simulation using different assumptions about the magnetopause reaction to solar wind variations. A substantial difference in the results of these numerical runs has been found in some intervals. For exam-
Table 1. Mean values of upstream solar wind parameters obtained from ACE for the four intervals. Here, $M_{f}=V / V_{f}$ is the fast Mach number, $M_{A}=V / V_{A}$ is the Alfvén Mach number $\left(V_{f}=\left(V_{s}^{2}+V_{A}^{2}\right)^{1 / 2}, V_{s}=(\gamma p / \rho)^{1 / 2}\right)$.

\begin{tabular}{cllll}
\hline Date & $M_{f}$ & $M_{A}$ & $\beta$ & $B_{z}$ GSM, nT \\
\hline 12 Feb 2001 & 6.8 & 8.3 & 0.57 & 2.4 \\
16 Dec 2001 & 5.0 & 6.1 & 0.60 & 6.5 \\
19 Dec 2001 & 6.0 & 6.5 & 0.20 & -1.6 \\
17 May 2002 & 6.7 & 7.8 & 0.41 & -0.3 \\
\hline
\end{tabular}

ple, the runs with a moving magnetopause predict the bow shock crossing on 12 February 2001 even a little later than it is observed (and later than it is predicted by the run with a fixed magnetopause). Correspondingly, average values of the MHD parameters in the magnetosheath predicted by the runs may differ in tens of percent. Generally the observed values are placed between the numerical results of the different runs. We do not show all the results in the paper because the main object of this work is the behaviour of the temperature anisotropy rather than a correct determination of the magnetopause shape in every particular case.

Another type of discrepancy between simulations and observations can be related to spatial structures in the solar wind. An additional time shift of several minutes is often required in order to match the simulations and observations of abrupt jumps in the magnetic field components. These are explained by variations of the IMF discontinuity orientation in the solar wind. Since the solar wind monitor near the Lagrangian point is relatively far from the Sun-Earth line (more than $25 R_{E}$ ), a variable slope of the IMF discontinuities would result in premature or delayed observations near the Sun-Earth line (e.g. Weimer et al., 2002). Moreover, the velocity of the solar wind varies over the whole time interval, and the discontinuities usually propagate with respect to the solar wind flow in the upstream or downstream direction: this may lead to an additional time shift near the Earth.

The second interval (Fig. 3) is an outward partial crossing of the magnetosheath on 16 December 2001. This case is characterized by a strong increase in the density between 05:30 and 07:10 UT observed one hour earlier in the supersonic solar wind. At the same time, $|V|$ is about $20-30$ percent larger, $\beta_{\|}$(in Fig. 7) is larger and $T_{\perp} / T_{\|}$is smaller than the surrounding values. The discrepancy between the predictions and the observations of the density in this interval is larger than the discrepancy of other parameters. The density enhancement is considerably underestimated by the model. The predicted $T_{\perp} / T_{\|}$is a little higher than the observed one only between 05:00 and 07:00 UT. The behavior of magnetic field components and $|V|$ is mainly predicted by the model.

Cluster seems to be in the outer magnetosheath in the third interval, on 19 December 2001, shown in Fig. 4. The 
previous studies of wave turbulence at electron scales (Mangeney et al., 2006; Lacombe et al., 2006) reveal that this is a rather quiet case with a low solar wind density. The numerical predictions underestimate the average $T_{\perp} / T_{\|}$and $\boldsymbol{B}$ over the interval. The density and velocity are well predicted in this case, except for some local variations.

The last interval on 17 May 2002, shown in Fig. 5, is again an outward crossing. At the beginning of the interval, Cluster seems to be near the magnetopause, which is confirmed by a high observed $T_{\perp} / T_{\|}$and a low $\beta$. Both the density and $|B|$ are systematically underestimated by the model, while average values of $T_{\perp} / T_{\|}$and $|V|$ are generally well predicted.

We have to raise here another question: How does the magnetic reconnection at the magnetopause influence the magnetosheath flow? Samsonov (2006) studied this problem with an isotropic MHD model using the same numerical method and varying boundary conditions at the inner boundary (assuming a non-zero normal velocity and magnetic field components). Generally this model gives a higher beta near the magnetopause and, correspondingly, a smaller temperature anisotropy. However, we do not think that the magnetic reconnection would be important in the four considered intervals, for which $X_{\mathrm{GSE}}$ is generally positive (see Fig. 1). We determine the mean values of the IMF $B_{z}$ component (in Table 1) and find that a prolonged period of a small negative $B_{z}$ has been observed by ACE only on 19 December 2001. But in this case Cluster was in the outer magnetosheath where the magnetopause reconnection should not influence the plasma flow. For the three other events, only relatively short intervals with negative $B_{z}$ have been observed. However, some local structures, like those observed in first $30 \mathrm{~min}$ of the interval on 12 February 2001, may be affected by the reconnection and these are not taken into account correctly by our present model.

Summarizing the predictions of the 3-D MHD model for $T_{\perp} / T_{\|}$in the four intervals, we conclude that the average predicted anisotropy is generally equal to or a little smaller than the observed one. The only exception is the strong density increase in the middle of the second interval, when the predicted anisotropy is larger (or, more precisely, the observed decrease in the anisotropy is underestimated in the simulation).

Our numerical model combines the double-adiabatic (CGL) MHD description of the plasma flow with the thresholds of the proton-cyclotron and mirror instabilities obtained from the linear kinetic theory. What happens if we simulate the anisotropy $T_{\perp} / T_{\|}$predicted by the CGL equations without the limitation by the two thresholds? Estimations (not shown) for the four considered intervals and results of other simulations (e.g. Samsonov and Pudovkin, 2000) show that $T_{\perp} / T_{\|}$in the magnetosheath can reach values from 3 up to 10 or more (the exception would be downstream of the parallel bow shock). This is reasonable because the CGL relations imply that $T_{\perp} / T_{\|} \sim B^{3} / n^{2}$.
Thus the observed temperature anisotropy shown in Figs. 2 to 5 is smaller than the pure CGL model predictions, but it is sometimes larger than the anisotropy predicted by our numerical model with the two thresholds. In the first part of this section, we have seen that the accuracy of the local MHD model can be less good in the magnetosheath flanks. But this last discrepancy can also be due to the fact that the thresholds (Eqs. 9 and 10) do not limit well the observed anisotropy. In the next section, we shall compare the observed anisotropy $T_{\perp p} / T_{\| p}$ with the theoretical anisotropy thresholds which depend only on the locally observed $\beta_{p}$.

\section{Role of proton-cyclotron and mirror instabilities}

Both the proton-cyclotron and mirror instabilities may grow in the magnetosheath, owing to a high proton temperature anisotropy. Since these instabilities have the same source of energy, they would be in competition: the growth of one instability results in a decrease in the temperature anisotropy and thus suppresses the growth of the other one. According to results of the linear kinetic theory (Gary et al., 1993) and of the hybrid simulation (McKean et al., 1994), the protoncyclotron instability may dominate for low- $\beta$ conditions with $\beta_{\| p} \lesssim 1$, and the mirror instability may dominate for high$\beta$ conditions with $\beta_{\| p} \gtrsim 6$. The 2-D hybrid simulations of McKean et al. (1994) show that both modes would grow for an intermediate beta (there $\beta_{\| p}=4$ ) and results of the mode competition depend on the percentage of solar wind $\alpha$ particles (Price et al., 1986). McKean et al. (1994) described a simulation run with the mode competition when the protoncyclotron instability may be responsible for only half of the magnetic fluctuation energy, but be more efficient in the reduction of the proton anisotropy.

Figures 6-9 show temporal variations of $\beta_{\| p}$ and $T_{\perp p} / T_{\| p}$ for the considered four intervals. Black lines are the observed profiles, color lines on the lower panels correspond to the upper boundary of $T_{\perp p} / T_{\| p}$, calculated for the mirror and proton-cyclotron instabilities: the red lines represent the maximal anisotropy in the case when the mirror instability controls the plasma state. The blue lines give the maximal anisotropy in the case when the proton-cyclotron instability plays the dominant role. The solid lines give the anisotropy (Eqs. 9 and 10) calculated with the maximum growth rate $\gamma_{m} / \Omega_{p}=10^{-2}$, the dashed lines correspond to $\gamma_{m} / \Omega_{p}=10^{-4}$ (the equations are in the caption of Fig. 6). As we will see the observed $T_{\perp p} / T_{\| p}$ fits well the temperature anisotropy calculated with the maximum growth rate within the range $\gamma_{m} / \Omega_{p} \in\left(10^{-4}, 10^{-2}\right)$.

According to Samsonov et al. (2001), the mirror threshold is valid for $1.0 \leq \beta_{\| p} \leq 10.0$ and $T_{e} / T_{\| p}=0.25$, the protoncyclotron threshold is valid for $0.01 \leq \beta_{\| p} \leq 10.0$, independent of $T_{e} / T_{\| p}$. Note that the observed $\beta_{\| p}$ is sometimes less than 1 , so that the predictions of the mirror instability threshold may be wrong there. Nevertheless the accuracy seems to 

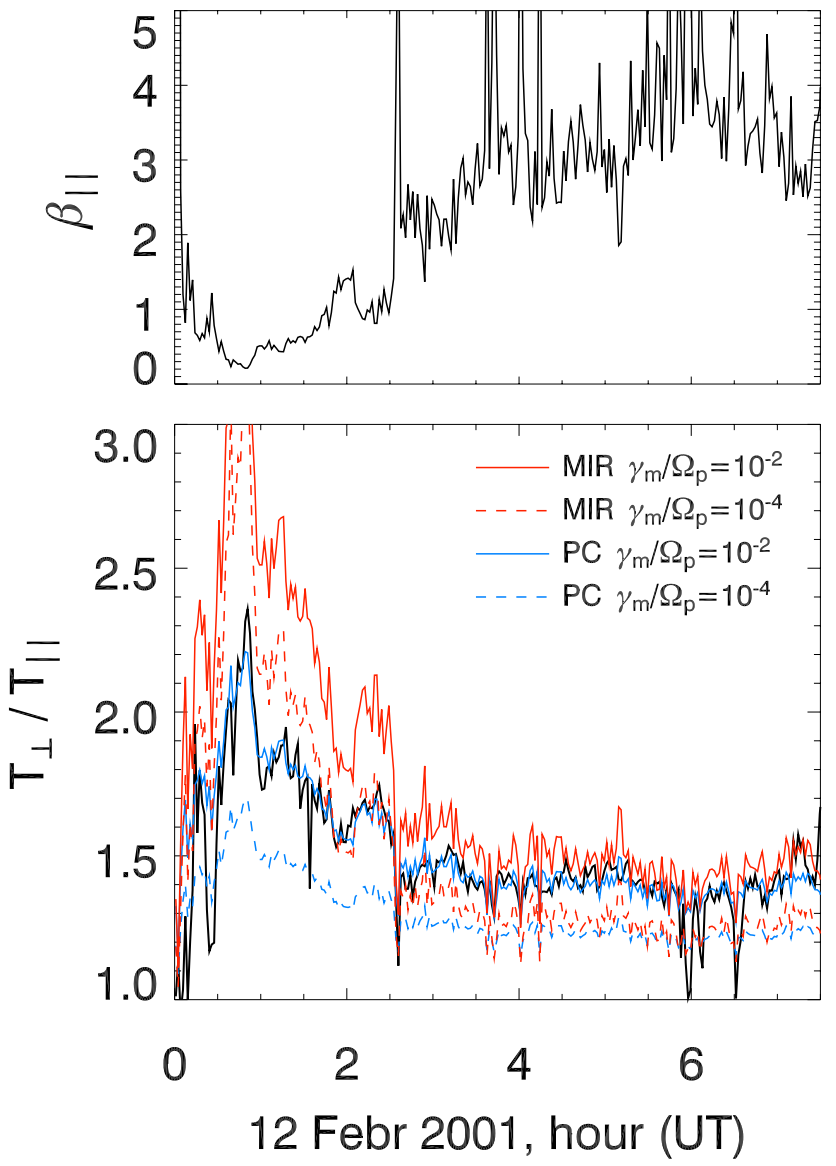

Fig. 6. Temporal variations of $\beta_{\| p}$ and of $T_{\perp p} / T_{\| p}$ in the first interval. The data are shown by black lines, the thresholds of the mirror instability found from the linear kinetic theory with $\gamma_{m} / \Omega_{p}=10^{-2}\left(T_{\perp p} / T_{\| p}=1+0.99 \beta_{\|}-0.63\right)$ and with $\gamma_{m} / \Omega_{p}=10^{-4}\left(T_{\perp p} / T_{\| p}=1+0.67 \beta_{\|}{ }^{-0.78}\right)$ are shown by solid red and dashed red lines, respectively, the thresholds of the proton-cyclotron instability found from the linear kinetic theory with $\gamma_{m} / \Omega_{p}=10^{-2}\left(T_{\perp p} / T_{\| p}=1+0.64 \beta_{\|}-0.41\right)$ and with $\gamma_{m} / \Omega_{p}=10^{-4}\left(T_{\perp p} / T_{\| p}=1+0.37 \beta_{\|}{ }^{-0.41}\right)$ are shown by solid blue and dashed blue lines, respectively.

be saved because Hellinger et al. (2006) obtained a similar threshold for the mirror mode for a smaller $\beta_{\| p}(\lesssim 0.1)$. The observed ratio $T_{e} / T_{\| p}$ is between 0.1 and 0.3 for the considered intervals.

Assuming the presence of a minor component of cold electrons, Hasegawa (1969) derived the marginal stability condition for the mirror mode $T_{\perp p} / T_{\| p}=1+1 / \beta_{\perp}$. We compare the temporal profiles of $T_{\perp p} / T_{\| p}$ obtained by the Hasegawa's condition (not shown) with the profiles of the mirror threshold for different $\gamma_{m}$ shown by the red lines in Figs. 6-9. We find that the traditional marginal stability threshold $T_{\perp p} / T_{\| p}=1+1 / \beta_{\perp}$ nearly coincides with or is slightly lower than the kinetic mirror threshold $T_{\perp p} / T_{\| p}=1+0.67 \beta_{\| p}{ }^{-0.78}$ obtained by Samsonov et al. (2001) for the maximum growth rate $\gamma_{m} / \Omega_{p}=10^{-4}$.
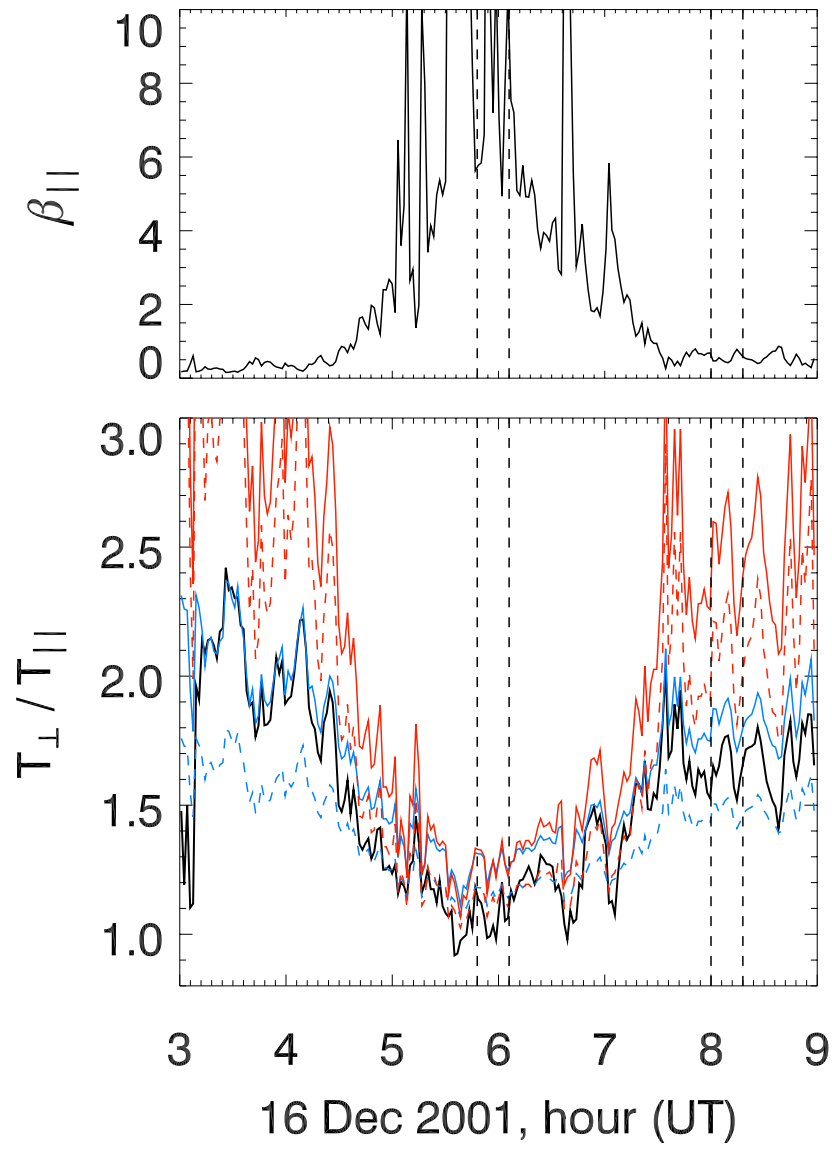

Fig. 7. The same parameters as in Fig. 6 in the second interval. The vertical dashed lines mark two 18-min intervals used for the spectral analysis in Sect. 6.

Looking at the first interval (Fig. 6), we see that the threshold of the proton-cyclotron instability with $\gamma_{m} / \Omega_{p}=10^{-2}$ agrees rather well with the observed temperature anisotropy over the whole interval. There is a low $\beta_{\| p}$ region nearly from the beginning to 02:32 UT which may correspond to the PDL (see Sect. 4). The threshold of the mirror instability with $\gamma_{m} / \Omega_{p}=10^{-2}$ is above the observed $T_{\perp p} / T_{\| p}$ over the whole interval. The threshold of the mirror instability with $\gamma_{m} / \Omega_{p}=10^{-4}$ is generally above the observed anisotropy before 02:32 UT and below it after. The threshold of the proton-cyclotron instability with $\gamma_{m} / \Omega_{p}=10^{-4}$ is everywhere below the observed $T_{\perp p} / T_{\| p}$.

The second interval (Fig. 7) may also be divided into two parts, with low and high $\beta_{\| p}$. In the low $\beta_{\| p}$ region before 04:30 UT, the proton-cyclotron threshold with $\gamma_{m} / \Omega_{p}=10^{-2}$ corresponds well to the observations. The high $\beta_{\| p}$ region is connected with the increase in the solar wind density discussed in the previous section. In this region, both the proton-cyclotron and mirror thresholds with $\gamma_{m} / \Omega_{p}=10^{-2}$ are above the observed anisotropy. The observed $T_{\perp p} / T_{\| p}$ is described better by the kinetic 

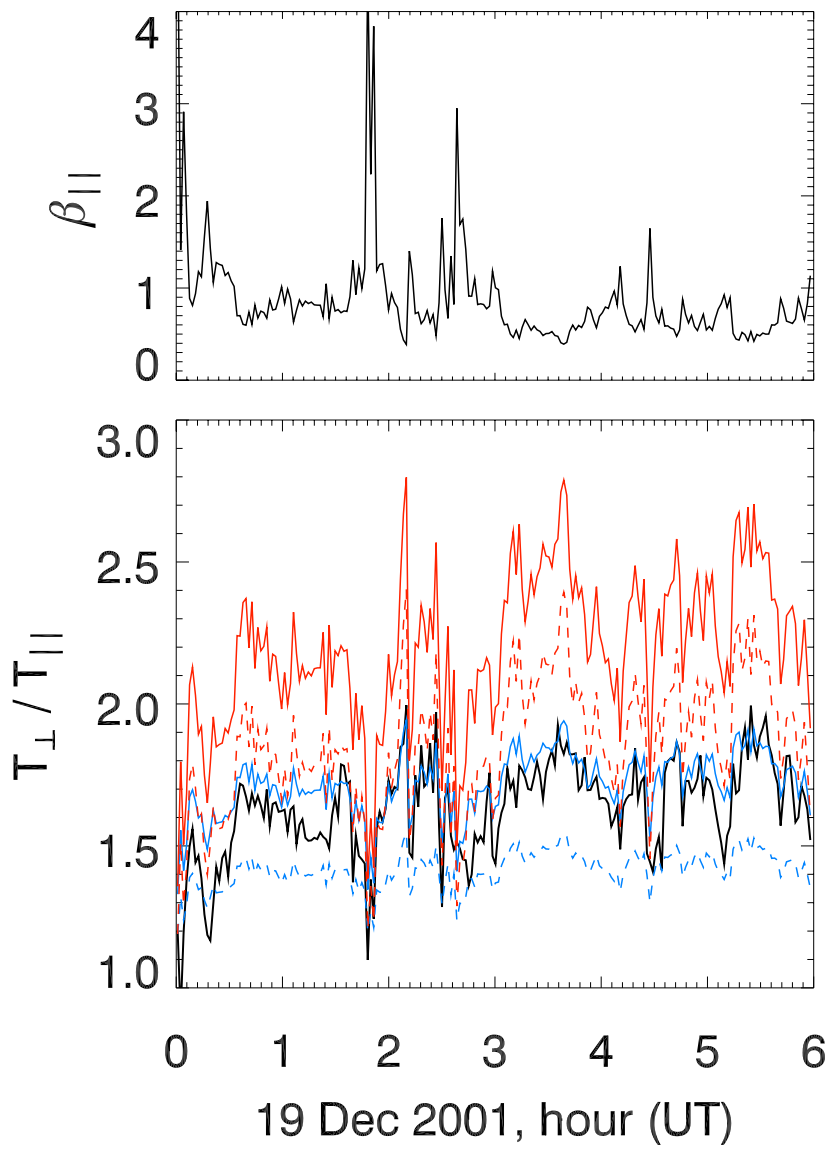

Fig. 8. The same parameters as in Fig. 6 in the third interval.

proton-cyclotron and mirror thresholds with $\gamma_{m} / \Omega_{p}=10^{-4}$, but occasionally the observed anisotropy is even below these thresholds, indicating that the magnetosheath plasma is stable with respect to the proton-cyclotron and mirror instabilities. The two 18-min intervals separated by vertical dashed lines in Fig. 7 are used for the spectral analysis in Sect. 6.

The observed $T_{\perp p} / T_{\| p}$ coincides with or is sometimes slightly below the proton-cyclotron threshold with $\gamma_{m} / \Omega_{p}=10^{-2}$ in the third interval in Fig. 8. The mirror thresholds with $\gamma_{m} / \Omega_{p}=10^{-2}$ and even $\gamma_{m} / \Omega_{p}=10^{-4}$ are above the measured $T_{\perp p} / T_{\| p}$ : this means that the role of the mirror waves should be negligible in this case.

The last interval in Fig. 9 consists of the two wellseparated parts with low and high $\beta_{\| p}$. Again, the protoncyclotron threshold with $\gamma_{m} / \Omega_{p}=10^{-2}$ agrees well with the observed $T_{\perp p} / T_{\| p}$ before 09:40 UT when $\beta_{\| p}$ is about 1 . Later, $\beta_{\| p}$ is larger than 2 and the observed $T_{\perp p} / T_{\| p}$ is between the thresholds with $\gamma_{m} / \Omega_{p}=10^{-2}$ and $\gamma_{m} / \Omega_{p}=10^{-4}$ of both instabilities. Therefore, a mixture of the two modes may exist. We shall present the observed fluctuation modes in Sect. 6.

In Fig. 10, we show the dependence of $T_{\perp p} / T_{\| p}$ on $\beta_{\| p}$, combining all the measurements in the four intervals
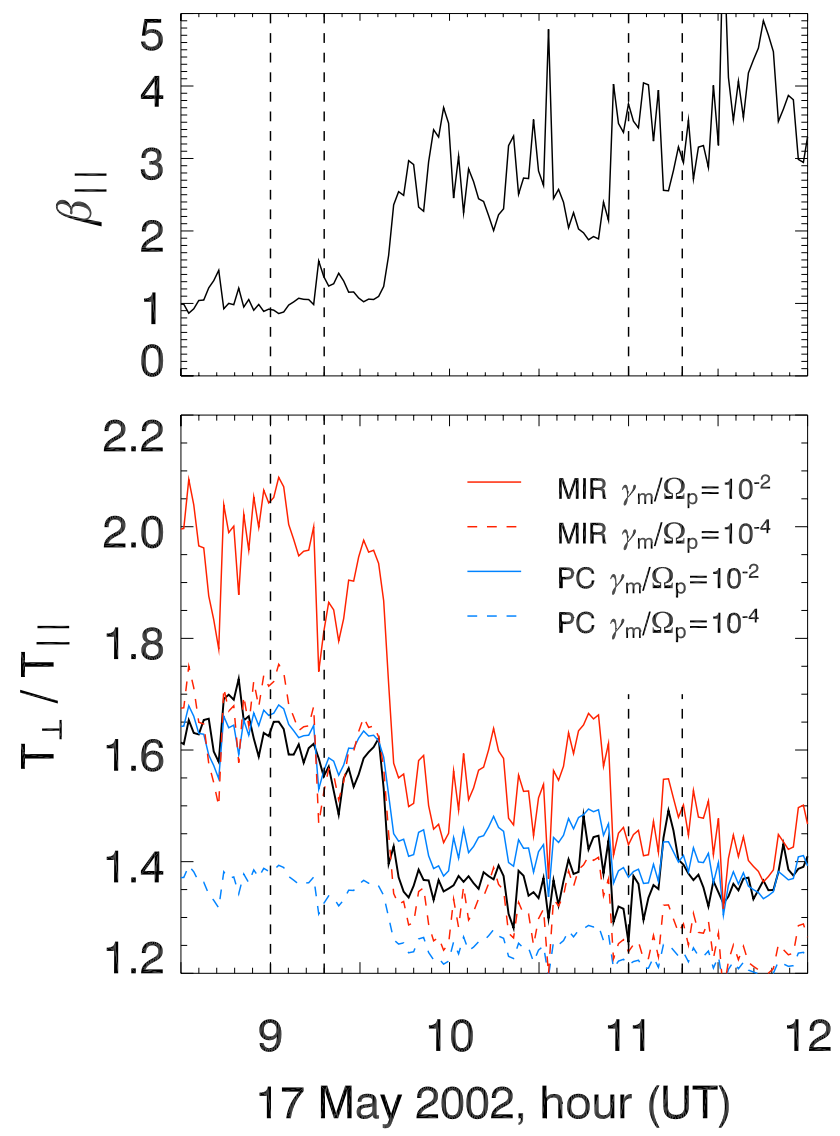

Fig. 9. The same parameters as in Fig. 6 in the fourth interval. The vertical dashed lines mark two 18-min intervals used for the spectral analysis in Sect. 6.

with the above-mentioned thresholds of the proton-cyclotron and mirror modes with $\gamma_{m} / \Omega_{p}=10^{-2}$ and $\gamma_{m} / \Omega_{p}=10^{-4}$. The proton-cyclotron threshold with a larger maximum growth rate $\gamma_{m} / \Omega_{p}=2.5 \times 10^{-2}$ is shown by a dash-dot blue line because it sometimes determines better an upper level of the observed anisotropy. The mirror threshold with $\gamma_{m} / \Omega_{p}=2.5 \times 10^{-2}$ (dash-dot red line) is well above the observed $T_{\perp p} / T_{\| p}$. The observations may be separated into two parts, one with $\beta_{\| p}<1$ and one with $\beta_{\| p}>2$. It appears that most points of $T_{\perp p} / T_{\| p}$ in the low- $\beta$ region are located between the two proton-cyclotron thresholds with $\gamma_{m} / \Omega_{p}=2.5 \times 10^{-2}$ and $\gamma_{m} / \Omega_{p}=10^{-4}$, but below the mirror thresholds. This confirms the previous works (see Anderson et al., 1994) where it has been shown that protoncyclotron-like fluctuations arise preferentially in the low- $\beta$ high-anisotropy plasma. In the high- $\beta$ region the protoncyclotron and the mirror thresholds with $\gamma_{m} / \Omega_{p}=10^{-2}$ are close enough, so that it is difficult to say what instability will dominate. This will be illustrated in the next section, where we consider the magnetic fluctuations observed by Cluster in the frequency range where the proton cyclotron and mirror mode are generally found. 


\section{Observed fluctuation modes}

We look at the properties of the magnetic fluctuations observed in regions with different values of $\beta_{\| p}$ and $A$. An analysis of the high resolution profile of the magnetic field components with the Morlet wavelets (Torrence and Compo, 1998), in intervals lasting $18 \mathrm{~min}$, yields average spectra between $0.003 \mathrm{~Hz}$ and $10 \mathrm{~Hz}$. We consider the spectral density of the parallel (or mirror-like) fluctuations $W_{\|}^{2}$ (fluctuations of the modulus of $B$ ), and the spectral density of the transverse (or Alfvénic) fluctuations $W_{\perp}^{2}=W_{t}^{2}-W_{\|}^{2}$, where $W_{t}^{2}=W_{x}^{2}+W_{y}^{2}+W_{z}^{2}$ is the sum of the intensity of the fluctuations in three directions.

In the spectra of Fig. 11, $W_{\|}^{2}$ (dashed line) and $W_{\perp}^{2}$ (solid line) are shown in two relatively low- $\beta_{\|} p$ regions (left column) and in two higher- $\beta_{\| p}$ regions (right column). The selected intervals corresponding to the spectra in the upper panels are displayed between vertical dashed lines in Fig. 7 (16 December 2001), and those corresponding to the lower panels are in Fig. 9 (17 May 2002).

In Fig. 11a $\left(\left\langle\beta_{\| p}\right\rangle=0.8,\langle A\rangle-1=0.7\right)$, there are some compressive fluctuations, but transverse fluctuations are everywhere dominant. The corresponding $T_{\perp p} / T_{\| p}$ profile (Fig. 7) indeed favours the growth of proton-cyclotron waves. (A large maximum in the vicinity of $f_{c i}$, around $0.4 \mathrm{~Hz}$, is a sign of Alfvén vortices recently discovered in the magnetosheath by Alexandrova et al. (2006). The relation between the linear proton-cyclotron instability and such nonlinear Alfvénic structures is the subject of a future work.) In Fig. $11 \mathrm{~b}\left(\left\langle\beta_{\| p}\right\rangle=9.8,\langle A\rangle-1=0.1\right)$, there are relatively much more compressive fluctuations than in Fig. 11a, on the same day. This is consistent with the results of Anderson et al. (1994), who show that a large $\beta_{\| p}$ favours the growth of compressive waves. However, the Alfvénic fluctuations are slightly dominant at every frequency in Fig. 11b, and this has not been observed by Anderson et al. (1994) at such high values of $\beta_{\| p}$. Let us recall that the results of Anderson et al. (1994) have been obtained with AMPTE/CCE (apogee $8.8 R_{E}$ ), i.e. for a highly compressed magnetosphere.

In Fig. 11c $\left(\left\langle\beta_{\| p}\right\rangle=1.1,\langle A\rangle-1=0.6\right)$, there is a peak of Alfvénic fluctuations around $0.1 \mathrm{~Hz}$, but there is a stronger peak of mirror-like fluctuations at $0.08 \mathrm{~Hz}$, in spite of the fact that the observed $T_{\perp p} / T_{\| p}$ (Fig. 9) is below the mirror mode threshold for $\gamma_{m} / \Omega_{p}=10^{-4}$. In Fig. $11 \mathrm{~d}\left(\left\langle\beta_{\| p}\right\rangle=3.4\right.$, $\langle A\rangle-1=0.4)$, the mirror fluctuations are not more intense than in Fig. 11c, in spite of the stronger $\beta_{\| p}$. Note that the mixture of the Alfvénic and mirror-like fluctuations observed here is in agreement with the results of Fig. 10 and with the simulations of McKean (1994) and of Hellinger and Trávníček (2005).

A systematic comparison of the ratio between $W_{\perp}^{2}$ and $W_{\|}^{2}$, at different frequencies, as a function of $\beta_{\| p}$ and $A$ is out of the scope of the present paper; it will be performed in the future. With the spectra of Fig. 11, we confirm that $\beta_{\| p}$ and

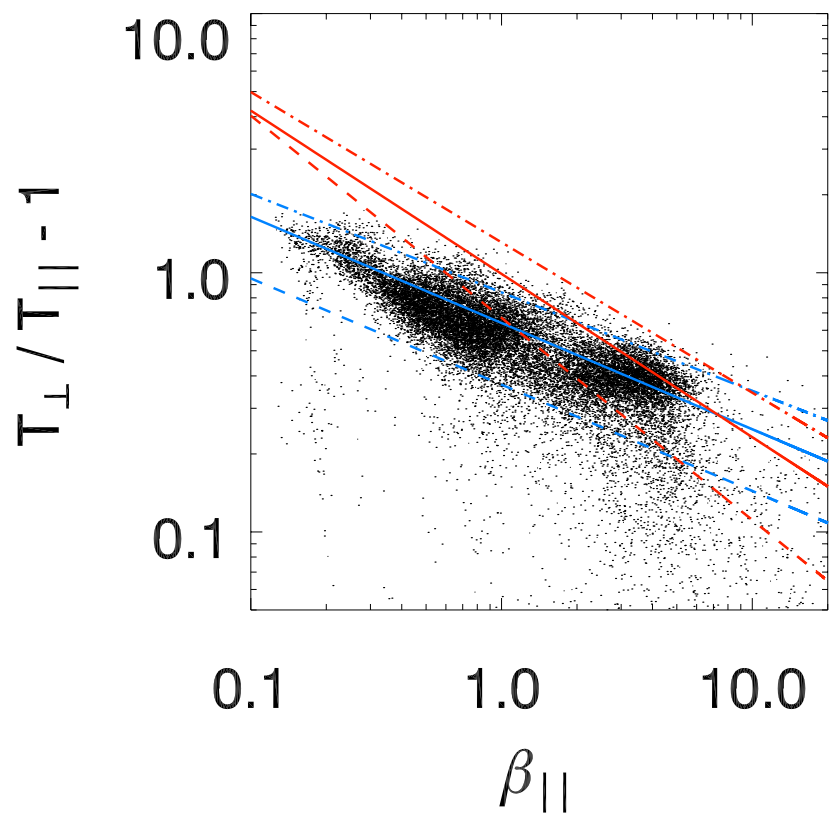

Fig. 10. Dependence of $T_{\perp p} / T_{\| p}$ on $\beta_{\| p}$. Black points show all measurements in the four intervals, red and blue lines are the same mirror and proton-cyclotron thresholds as those shown in Figs. 6-9. The proton-cyclotron threshold $T_{\perp p} / T_{\| p}=1+0.84 \beta_{\|}-0.38$ and the mirror threshold $T_{\perp p} / T_{\| p}=1+1.31 \beta_{\|}{ }^{-0.58}$ corresponding to the maximum growth rate $\gamma_{m} / \Omega_{p}=2.5 \times 10^{-2}$ are shown by blue and red dash-dot lines, respectively.

$A=T_{\perp p} / T_{\| p}$ are important parameters for the determination of the dominant wave mode, but other parameters certainly play a part. And one of them is the percentage of $\alpha$ particles (e.g. Price et al., 1986; Hubert et al., 1998).

During the four considered intervals, the number $n_{\alpha}$ of $\alpha$ particles is given by the CIS1(CODIF) instrument. The magnetosheath contains $n_{\alpha} / n_{p}=2$ to $4 \%$ on the 12 February 2001,2 to $5 \%$ on 16 December 2001,5 to $9 \%$ on 19 December 2001 and 4 to $7 \%$ on 17 May 2002 (Jean-Michel Bosqued, private communication, 2007). According to Gary et al. (1994b), a percentage of 4 to 5\% of $\alpha$ particles allows the growth of helium-cyclotron waves, but contributes to the damping of the proton-cyclotron mode, thus raising its threshold. According to Fig. 3 of Hellinger and Trávníček (2005), the growth of the proton-cyclotron mode is indeed reduced by the presence of $5 \%$ of $\alpha$ particles, mainly for $\beta_{\| p} \simeq 0.1$ but also up to $\beta_{\| p} \simeq 1$ to 3 . At such $\beta_{\| p}$, the presence of $\alpha$ particles could thus favour the mirror mode, as observed in Fig. 11c.

The conclusion which can be drawn from Figs. 10 and 11 is that the Alfvénic fluctuations dominate the magnetic spectrum for $\beta<1$, in agreement with the previous works. However, we observe that above $\beta=1$ both types of magnetic fluctuations seem to control the quasi-linear equilibrium of the magnetosheath state. 

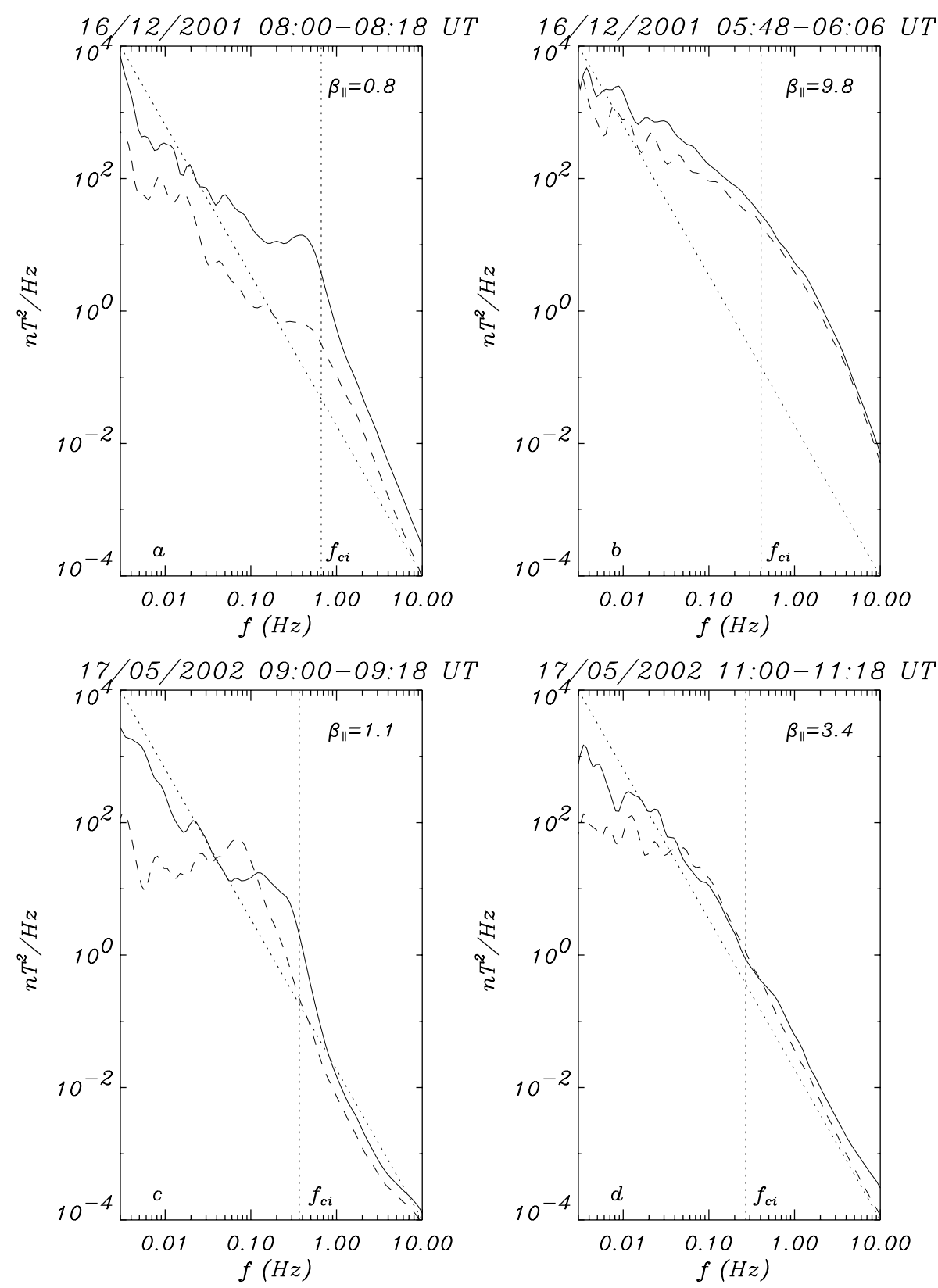

Fig. 11. Solid lines: average spectra $W_{\perp}^{2}$ of the transverse (Alfvénic) magnetic fluctuations. Dashed lines: average spectra $W_{\|}^{2}$ of the compressive (mirror) magnetic fluctuations, for different intervals lasting $18 \mathrm{~min}$. The dotted vertical line gives the average value of the proton cyclotron frequency for the considered interval. The dotted diagonal helps to see the variations of the spectral density for a given day and from day to day.

\section{Conclusion}

We have simulated four magnetosheath intervals measured by the Cluster spacecraft, using the numerical 3-D doubleadiabatic MHD model and taking upper limits of the predicted proton temperature anisotropy from the kinetic thresholds of the proton-cyclotron and mirror instabilities (Samsonov et al., 2001). The ACE magnetic field and plasma solar wind data have been used as input parameters of the model.
We assume that the magnetopause is a fixed and impenetrable boundary, i.e. without magnetic reconnection. Generally the numerical predictions agree with the observations, however, the accuracy of the predictions for these crossings on the magnetosheath flanks is worse than that obtained by similar models in the subsolar region. We think that the problem may be caused by an inaccurate approximation of the magnetopause shape in the local magnetosheath model. Therefore, sometimes the average values of the predicted density and 
magnetic field differ from the observed ones. Another discrepancy between the predictions and observations is connected with the time of arrival of solar wind discontinuities from the Lagrangian point to the magnetosheath. Since the orientation of the IMF discontinuities, their speed in the plasma frame and the solar wind velocity in a time interval may vary, additional time shifts from one minute to tens minutes are often required.

The predicted temperature anisotropy $T_{\perp p} / T_{\| p}$ in the four intervals is equal to or slightly smaller than the observed one. In Figs. 6-9, we compare the observed temperature anisotropy with the theoretical kinetic thresholds used in our MHD modelling. In every interval we can separate low- $\beta$ high $T_{\perp p} / T_{\| p}$ regions (with $\beta_{\| p} \lesssim 1$ ), where the observed ratio $T_{\perp p} / T_{\| p}$ is described well by the kinetic threshold of the proton-cyclotron instability and it is below the thresholds of the mirror instability. In high- $\beta-$ low $T_{\perp p} / T_{\| p}$ regions, the observed $T_{\perp p} / T_{\| p}$ is often between the proton-cyclotron and mirror thresholds (with the maximum growth rate varying from $\gamma_{m} / \Omega_{p}=10^{-4}$ to $2.5 \times 10^{-2}$ ), so one may assume that the growth rate of both instabilities could be nearly equal. There are only a few short intervals during the large density enhancement on 16 December, 2001 when the observed $T_{\perp p} / T_{\| p}$ is a little below the proton-cyclotron and mirror thresholds.

We also consider the well-known mirror marginal stability threshold $T_{\perp p} / T_{\| p}=1+1 / \beta_{\perp}$ and find that it generally coincides with or is slightly lower than the kinetic mirror threshold $T_{\perp p} / T_{\| p}=1+0.67 \beta_{\|}{ }^{-0.78}$ obtained by Samsonov et al. (2001) for the maximum growth rate $\gamma_{m} / \Omega_{p}=10^{-4}$.

We analyze several magnetic field power spectra between $0.003 \mathrm{~Hz}$ and $10 \mathrm{~Hz}$ both in low- $\beta$ and in high- $\beta$ regions. This analysis shows that the values of $\beta_{\| p}$ and $T_{\perp p} / T_{\| p}$ are important but not the only parameters which would determine the dominant mode, compressive or transverse (i.e. mirror or Alfvénic) fluctuations at the proton scales in the magnetosheath. Other factors, such as, for example, the percentage of $\alpha$ particles in the solar wind, may also be rather important. For $\beta_{\| p}$ from 1 to 10 , which is typical for the magnetosheath (except the PDL), there would be usually a mixture of compressive and transverse fluctuations, without a certain dominance of one of them. Even when the threshold of the proton-cyclotron instability with the growth rate $\gamma_{m} / \Omega_{p} \simeq 10^{-2}$ determines well the observed temperature anisotropy, while the growth rate $\gamma_{m} / \Omega_{p}$ of the mirror instability is less than $10^{-4}$, nevertheless, the wave power spectrum may display compressive mirror-like fluctuations, together with the transverse Alfvénic fluctuations.

Summarizing all obtained results, we can conclude that the bounded anisotropy model (Denton et al., 1994), using two separate kinetic thresholds for the proton-cyclotron and mirror instabilities (Samsonov et al., 2001), can describe rather well the proton temperature anisotropy in the flank magnetosheath.
Acknowledgements. The authors thank J.-M. Bosqued for providing Cluster proton data, A. Balogh and E. A. Lucek for the high resolution magnetic field data, and the ACE MAG and SWEPAM instrument teams and the ACE Science Center for providing the ACE data. The work of A. A. Samsonov was supported by INTAS grant 03-55-1034 for young scientists. O. Alexandrova acknowledges the support of French National Space Agency (CNES).

Topical Editor I. A. Daglis thanks S. A. Fuselier and another referee for their help in evaluating this paper.

\section{References}

Alexandrova, O., Mangeney, A., Maksimovic, M., Lacombe, C., Cornilleau-Wehrlin, N., Lucek, E. A., Décréau, P. M. E., Bosqued, J.-M., Travníček, P., and Fazakerley, A. N.: Cluster observations of finite amplitude Alfvén waves and small-scale magnetic filaments downstream of a quasi-perpendicular shock, J. Geophys. Res., 109(A18), 5207, doi:10.1029/2003JA010056, 2004.

Alexandrova, O., Mangeney, A., Maksimovic, M., CornilleauWehrlin, N., Bosqued, J.-M., and André, M.: Alfvén vortex filaments observed in magnetosheath downstream of a quasiperpendicular bow shock, J. Geophys. Res., 111(A10), 12208, doi:10.1029/2006JA011934, 2006.

Anderson, B. J. and Fuselier, S. A.: Magnetic pulsations from 0.1 to 4.0 Hz and associated plasma properties in the Earth's subsolar magnetosheath and plasma depletion layer, J. Geophys. Res., 98, 1461-1479, 1993.

Anderson, B. J., Fuselier, S. A., Gary, S. P., and Denton, R. E.: Magnetic spectral signatures in the Earth's magnetosheath and plasma depletion layer, J. Geophys. Res., 99, 5877-5891, 1994.

Balogh, A., Dunlop, M. W., Cowley, S. W. H., Southwood, D. J., Thomlinson, J. G., Glassmeier, K. H., Musmann, G., Lühr, H., Buchert, S., Acuña, M. H., Fairfield, D. H., Slavin, J. A., Riedler, W., Schwingenschuh, K., Kivelson, M. G., and the Cluster magnetometer team: The Cluster Magnetic Field Investigation, Space Sci. Rev., 79, 65-92, 1997.

Chew, G. F., Goldberger, M. L., and Low, F. E.: The Boltzmann equation and the one-fluid hydromagnetic equations in the absence of particle collisions, Proc. R. Soc. London Ser. A, 236, 112-118, 1956.

Crooker, N. U., Siscoe, G. L., and Geller, R. B.: Persistent pressure anisotropy in the subsonic magnetosheath region, Geophys. Res. Lett., 3, 65-68, 1976.

Czaykowska, A., Bauer, T. M., Treumann, R. A., and Baumjohann, W.: Mirror waves downstream of the quasi-perpendicular bow shock, J. Geophys. Res., 103, 4747-4753, 1998.

Czaykowska, A., Bauer, T. M., Treumann, R. A., and Baumjohann, W.: Magnetic field fluctuations across the Earth's bow shock, Ann. Geophys., 19, 275-287, 2001, http://www.ann-geophys.net/19/275/2001/.

Denton, R. E., Anderson, B. J., Gary, S. P., and Fuselier, S. A.: Bounded anisotropy fluid model for ion temperature, J. Geophys. Res., 99, 11 225-11 241, 1994.

Denton, R. E. and Lyon, J. G.: Density depletion in an anisotropic magnetosheath, Geophys. Res. Lett., 23, 2891-2894, 1996.

Erkaev, N. V., Farrugia, C. J., and Biernat, H. K.: Threedimensional, one-fluid, ideal MHD model of magnetosheath flow 
with anisotropic pressure, J. Geophys. Res., 104, 6877-6887, 1999.

Farrugia, C. J., Erkaev, N. V., Vogl, D. F., Biernat, H. K., Oieroset, M., Lin, R. P., and Lepping, R. P.: Anisotropic magnetosheath: Comparison of theory with Wind observations near the stagnation streamline, J. Geophys. Res., 106, 29373-29385, 2001.

Filbert, P. C. and Kellogg, P. J.: Electrostatic noise at the plasma frequency beyond the Earth's bow shock, J. Geophys. Res., 84, 1369-1381, 1979.

Fuselier, S. A., Anderson, B. J., Gary, S. P., and Denton, R. E.: Inverse correlations between the ion temperature anisotropy and plasma beta in the Earth's quasi-parallel magnetosheath, J. Geophys. Res., 99, 14 931-14 936, 1994.

Gary, S. P.: Theory of Space Plasma Microinstabilities, Cambridge University Press, New York, 1993.

Gary, S. P., Fuselier, S. A., and Anderson, B. J.: Ion anisotropy instabilities in the magnetosheath, J. Geophys. Res., 98, 14811488, 1993.

Gary, S. P., McKean, M. E., Winske, D., Anderson, B. J., Denton, R. E., and Fuselier, S. A.: The proton cyclotron instability and the anisotropy/ $\beta$ inverse correlation, J. Geophys. Res., 99, $5903-$ 5914, 1994a.

Gary, S. P., Convery, P. D., Denton, R. E., Fuselier, S. A., and Anderson, B. J.: Proton and helium cyclotron anisotropy instability thresholds in the magnetosheath, J. Geophys. Res., 99, 59155934, 1994b.

Hasegawa, A.: Drift mirror instability in the magnetosphere, Phys. Fluids, 12, 2642-2650, 1969.

Hellinger, P. and Trávníček, P.: Magnetosheath compression: Role of characteristic compression time, alpha particle abundance, and alpha/proton relative velocity, J. Geophys. Res., 110, A04210, doi:10.1029/2004JA010687, 2005.

Hellinger, P., Trávníček, P., Kasper, J. C., and Lazarus, A. J.: Solar wind proton temperature anisotropy: Linear theory and WIND/SWE observations, Geophys. Res. Lett., 33, L09101, doi:10.1029/2006GL025925, 2006.

Hubert, D., Harvey, C. C., and Russell, C. T.: Observations of magnetohydrodynamic modes in the Earth's magnetosheath at 0600 LT, J. Geophys. Res., 94, 17305-17309, 1989.

Hubert, D., Lacombe, C., Harvey, C. C., Moncuquet, M., Russell, C. T., and Thomsen, M. F.: Nature, properties and origin of low-frequency waves from an oblique shock to the inner magnetosheath, J. Geophys. Res., 103, 26 783-26798, 1998.

Lacombe, C. and Belmont, G.: Waves in the Earth's magnetosheath: Observations and interpretations, Adv. Space Res., 15, 329-340, 1995.

Lacombe, C., Samsonov, A. A., Mangeney, A., Maksimovic, M., Cornilleau-Wehrlin, N., Harvey, C. C., Bosqued, J.-M., and Travníček, P.: CLUSTER observations in the magnetosheath: 2 . Intensity of the turbulence at electron scales, Ann. Geophys., 24, 3523-3531, 2006, http://www.ann-geophys.net/24/3523/2006/.

Lucek, E. A., Dunlop, M. W., Horbury, T. S., Balogh, A., Brown, P., Cargill, P., Carr, C., Fornaçon, K. H., Georgescu, E., and Oddy, T.: Cluster magnetic field observations in the magnetosheath: four point measurements of mirror structures, Ann. Geophys., 19, 1421-1428, 2001, http://www.ann-geophys.net/19/1421/2001/.

Mangeney, A., Lacombe, C., Maksimovic, M., Samsonov, A. A.,
Cornilleau-Wehrlin, N., Harvey, C. C., Bosqued, J.-M., and Travníček, P.: CLUSTER observations in the magnetosheath: 1 . Anisotropies of the wave vector distribution of the turbulence at electron scales, Ann. Geophys., 24, 3507-3521, 2006, http://www.ann-geophys.net/24/3507/2006/.

McKean, M. E., Winske, D., and Gary, S. P.: Two-dimensional simulations of ion anisotropy instabilities in the magnetosheath, J. Geophys. Res., 99, 11 141-11 153, 1994.

Phan, T.-D., Paschmann, G., Baumjohann, W., Sckopke, N., and Lühr, H.: The magnetosheath region adjacent to the dayside magnetopause: AMPTE/IRM observations, J. Geophys. Res., 99, 121-141, 1994.

Phan, T.-D., Larson, D. E., Lin, R. P., et al.: The subsolar magnetosheath and magnetopause for high solar wind ram pressure: WIND observations, Geophys. Res. Lett., 23, 1279-1282, 1996.

Pokhotelov, O. A., Sagdeev, R. Z., Balikhin, M. A., and Treumann, R. A.: Mirror instability at finite ion-Larmor radius wavelengths, J. Geophys. Res., 109, A09213, doi:10.1029/2004JA010568, 2004.

Price, C. P., Swift, D. W., and Lee, L.-C.: Numerical simulation of nonoscillatory mirror waves at the Earth's magnetosheath, J. Geophys. Res., 91, 101-112, 1986.

Pudovkin, M. I., Besser, B. P., and Zaitseva, S. A.: Magnetopause stand-off distance in dependence on the magnetosheath and solar wind parameters, Ann. Geophys., 16, 388-396, 1998, http://www.ann-geophys.net/16/388/1998/.

Rème, H., Bosqued, J. M., Sauvaud, J. A., et al.: The Cluster Ion Spectrometry (CIS) Experiment, Space Sci. Rev., 79, 303-350, 1997.

Sahraoui, F., Pinçon, J. L., Belmont, G., Rezeau, L., CornilleauWehrlin, N., Robert, P., Mellul, L., Bosqued, J. M., Balogh, A., Canu, P., and Chanteur, G.: ULF wave identification in the magnetosheath: The k-filtering technique applied to Cluster II data, J. Geophys. Res., 108(A9), 1335, doi:10.1029/2002JA009587, 2003.

Samsonov, A. A. and Pudovkin, M. I.: Ideal anisotropic plasma flow around a sphere in the CGL-approach, Geomagnetism and aeronomy (in Russian), 38, 50-57, 1998.

Samsonov, A. A. and Pudovkin, M. I.: Application of the bounded anisotropy model for the dayside magnetosheath, J. Geophys. Res., 105, 12 859-12 867, 2000.

Samsonov, A. A., Pudovkin, M. I., Gary, S. P., and Hubert, D.: Anisotropic MHD model of the dayside magnetosheath downstream of the oblique bow shock, J. Geophys. Res., 106, 21689 $21699,2001$.

Samsonov A. A. and Meister, C.-V.: Anisotropic MHD model for the magnetosheath of Saturn, Planet. Space Sci., 50, 519-525, 2002.

Samsonov, A. A. and Hubert, D.: The steady-state slow shock inside the Earth's magnetosheath: to be or not to be? Part 2. Numerical 3-D MHD modeling, J. Geophys. Res., 109, A01218, doi:10.1029/2003JA010006, 2004.

Samsonov, A. A.: Numerical modeling of the Earth's magnetosheath for different IMF orientations, Adv. Space Res., 38(8), 1652-1656, doi:10.1016/j.asr.2005.06.009, 2006.

Samsonov, A. A., Nemecek, Z., and Safrankova, J.: Numerical MHD modeling of propagation of interplanetary shock through the magnetosheath, J. Geophys. Res., 111, A08210, doi:10.1029/2005JA011537, 2006. 
Schäfer, S., Glassmeier, K.-H., Narita, Y., Fornaçon, K. H., Dandouras, I., and Fränz, M.: Statistical phase propagation and dispersion analysis of low frequency waves in the magnetosheath, Ann. Geophys., 23, 3339-3349, 2005, http://www.ann-geophys.net/23/3339/2005/.

Shue, J.-H., Song, P., Russell, C. T., Steinberg, J. T., Chao, J. K., Zastenker, G., Vaisberg, O. L., Kokubun, S., Singer, H. J., Detman, T. R., and Kawano, H.: Magnetopause location under extreme solar wind conditions, J. Geophys. Res., 103, 17 691-17 700, 1998.

Sibeck, D. G., Lopez, R. E., and Roelof, E. C.: Solar wind control of the magnetopause shape, location and motion, J. Geophys. Res., 96, 5489-5495, 1991.

Song, P., Russell, C. T., and Gary, S. P.: Identification of lowfrequency fluctuations in the terrestrial magnetosheath, J. Geophys. Res., 99, 6011-6025, 1994.
Torrence, C. and Compo, G. P.: A Practical Guide to Wavelet Analysis., Bull. Am. Meteorol. Soc., 79, 61-78, 1998.

Tóth, G. and Odstrcil, D.: Comparison of some flux corrected transport and total variation diminishing numerical schemes for hydrodynamic and magnetohydrodynamic problems, J. Computat. Phys., 128, 82-100, 1996.

Tsurutani, B. T., Smith, E. J., Anderson, R. R., Ogilvie, K. W., Scudder, J. D., Baker, D. N., and Bame, S. J.: Lion roars and nonoscillatory drift mirror waves in the magnetosheath, J. Geophys. Res., 87, 6060-6072, 1982.

Weimer, D. R., Ober, D. M., Maynard, N. C., Burke, W. J., Collier, M. R., McComas, D. J., Ness, N. F., and Smith, C. W.: Variable time delays in the propagation of the interplanetary magnetic field, J. Geophys. Res., 107(A8), SMP 29, doi:10.1029/2001JA009102, 2002. 